\title{
DARK ENERGY AND NON-LINEAR PERTURBATIONS
}

\author{
C. VAN DE BRUCK \\ Department of Applied Mathematics \\ The University of Sheffield \\ Hounsfield Road \\ Sheffield S3 2RH \\ United Kingdom \\ D. F. MOTA \\ Astrophysics Department, University of Oxford \\ Keble Road, OX1 3RH, United Kingdom \\ and \\ Institute of Theoretical Astrophysics, University of Oslo \\ N-0315 Oslo, Norway
}

\begin{abstract}
Dark energy might have an influence on the formation of non-linear structures during the cosmic history. For example, in models in which dark energy couples to dark matter, it will be non-homogeneous and will influence on the collapse of a dark matter overdensity. We use the spherical collapse model to estimate how much influence dark energy might have.
\end{abstract}

\section{Introduction}

One of the most important goals of contemporary cosmology is to unreveal the properties of dark energy. This energy form is thought to be responsible for the observed accelerating expansion of the present day universe. There are several methods used to study the properties of dark energy. The important ones make use of the anisotropies in the Cosmic Microwave Background Radiation (CMB), the evolution of large scale structure formation (LSS) and/or the distances of high redshift supernovae (see e.g. the overview by Peebles and Ratra ${ }^{1}$ ).

In this contribution we address the question whether dark energy can have some impact on the formation of non-linear structures in the universe, such as clusters of galaxies or galaxies itself. In doing so, we assume that dark energy is a scalar field, pervading the universe. It will obviously 
depend on the properties of dark energy if this scalar field has any influence on non-linear structure formation. For example, if dark energy couples to dark matter, there is an extra force between the dark matter particles, mediated by dark energy. This possibility was first discussed in detail by Wetterich $^{2}$ and afterwards in particular by Amendola ${ }^{3}$. However, even if dark energy does not couple to dark matter, backreaction effects of the gravitational field might influence the evolution of dark energy inside a non-linear overdensity.

Here, we use the spherical collapse model to study the influence of dark energy on non-linear structure formation.
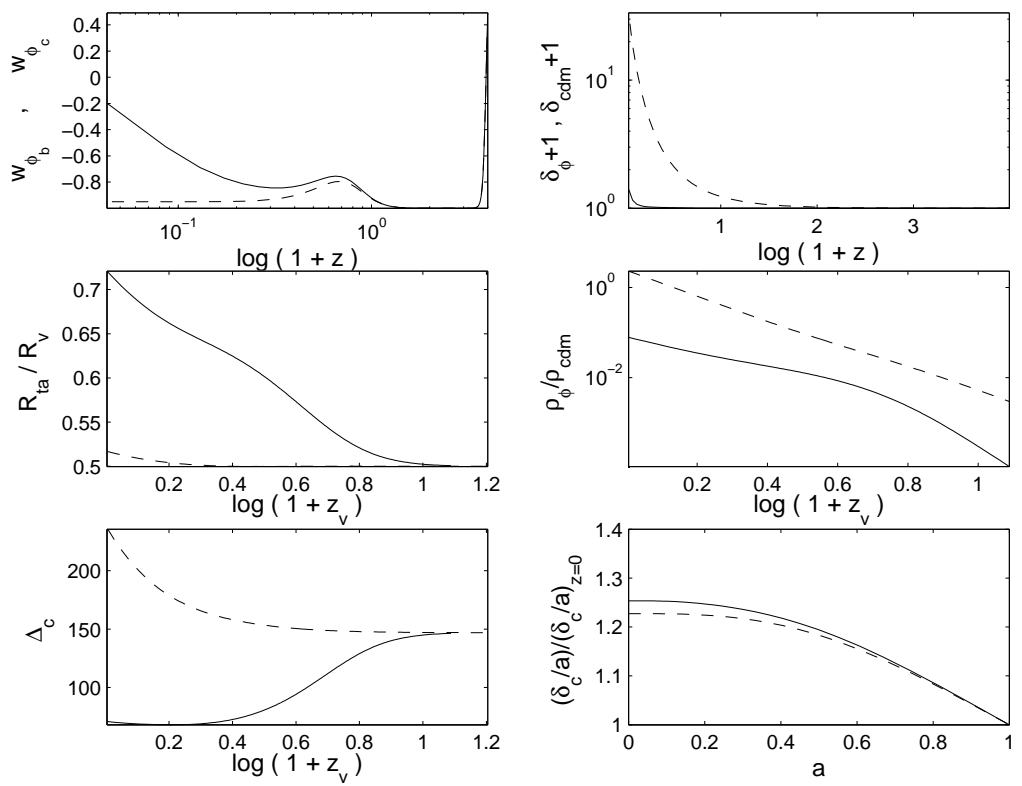

Figure 1. Double exponential potential (see text). Top left panel: Full collapse of dark energy: evolution of $w_{\phi}$ in the background (dashed line) and inside the overdensity (solid line) as a function of redshift. Top right panel: evolution of $\rho_{\phi, \text { overdensity }} / \rho_{\phi}$, outside (solid line) and $\rho_{\mathrm{cdm}}$,overdensity $/ \rho_{\mathrm{cdm}}$,outside as a function of redshift in the case of clustering of dark energy. Middle left panel: $R_{v} / R_{t}$ as a function of virialisation redshift in the case of homogeneous dark energy (dashed line) and collapsing dark energy (solid line). Middle right panel: The ratio $\rho_{\phi} / \rho_{\text {darkmatter }}$ inside the overdensity as a function of virialisation redshift. Bottom left: $\Delta_{c}$ as a function of virialisation redshift in the case of homogeneous (dashed line) and inhomogeneous (solid line) dark energy. Bottom right: the linear density contrast as a function of the scale factor $a$. 


\section{The spherical collapse model}

The spherical collapse model is based on the assumption that an overdensity can be treated as a homogeneous and isotropic, closed "sub-universe", embedded in our universe. For a cold dark matter universe, this assumption is justified by Birkhoff's theorem. However, as soon there is a second fluid, such as radiation, dark energy, etc., this (idealised) over-density can, and will, exchange energy with its surroundings. In the case of dark energy studied here, however, the exchange will only be a small fraction of the total energy, since dark energy is subdominant for most parts of the cosmic history. Therefore, it should be a not too bad description once the energy out- or inflow is specified. However, the spherical collapse model does not specify the energy outflow (which we denote by $\Gamma$ ) of dark energy into the surroundings of the overdensity. Therefore, we have to make assumptions about $\Gamma$. In our work ${ }^{4}$ we considered two extreme cases. In the first case, we assumed that dark energy does not cluster at all but is homogeneous throughout space. In the second case we assumed that it fully collapses along with dark matter. Clearly, the reality might be somewhere between these two possibilities. However, we will get an idea about the difference to be expected.

The typical time-evolution of a spherical overdensity is as follows: Initially, the overdensity expands with almost the same rate as the universe. However, since the density is higher, the expansion of the overdensity will eventually slow down until it starts to contract. The point at which the expansion turns into contraction is called turnaround. Without dissipation, the overdensity would collapse to a singularity. However, in reality, energies inside the overdensities viralise and the sphere ends up at some final radius (virialisation). In our calculations, we follow the evolution of the sphere until virialisation is reached. We have studied several different potentials and refer to our paper ${ }^{4}$ for the details of the calculation. Here, we consider only three different potentials as models for dark energy. The first one is a double exponential potential $^{5}$. The form is $V(\phi)=M(\exp (\alpha \phi)+\exp (\beta \phi))$. The second is the well known supergravity potential ${ }^{6}$, which is $V(\phi)=M \exp \left(\phi^{2}\right) / \phi^{\gamma}$. The third is a exponential potential with power-law modifications ${ }^{7}$, i.e. $V(\phi)=M\left(A+(\phi-B)^{2}\right) \exp (-\gamma \phi)$.

The results of our calculations can be found in Figs.1 - 3. Apart from the equation of state of dark energy inside and outside the overdensity and the density contrast of dark energy and dark matter, we calculate the ratio of 

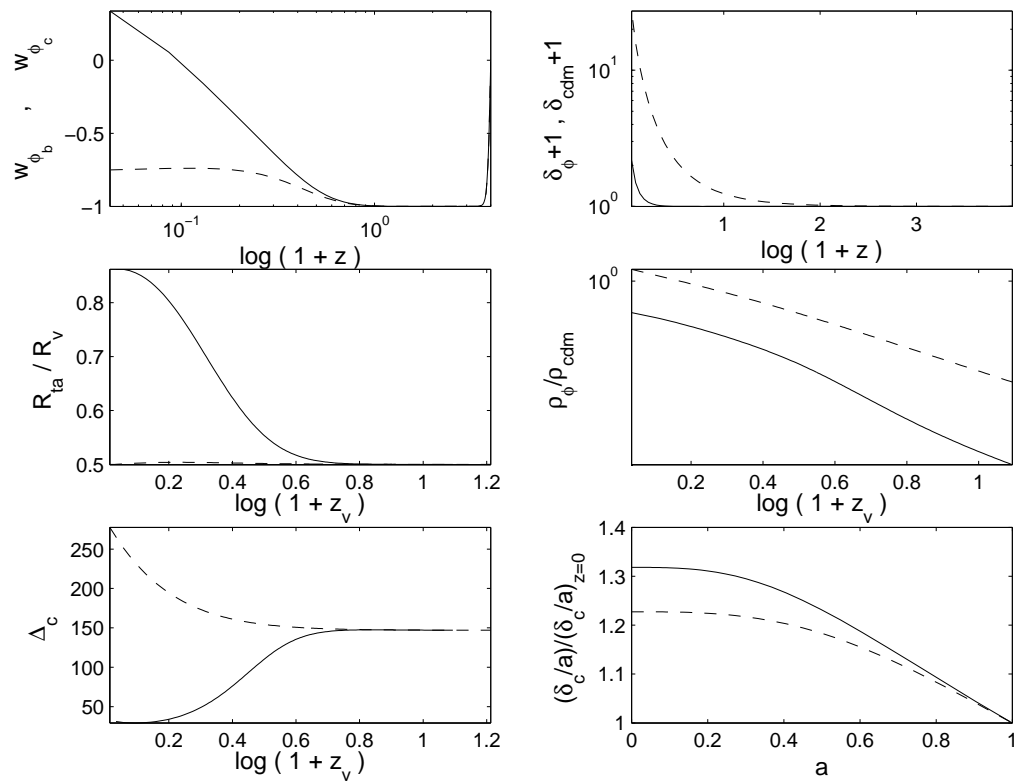

Figure 2. The same as Figure 1, but for the supergravity potential (see text).

the radius at turnaround $R_{t}$ to the radius at virialisation $R_{v}$ as a function of redshift at which the overdensity virialises $\left(z_{v}\right)$. The latter is equal to 0.5 in the case of the standard cold dark matter model and has a slight dependence on the cosmological constant in a $\Lambda \mathrm{CDM}$ model ${ }^{8}$. It can be seen from Figs. 1,2 and 3 that in the case of a homogeneous dark energy component the ratio $R_{t} / R_{v}$ depends on the model of dark energy, but is still of order 0.5. If dark energy collapses together with dark matter, this quantity depends strongly on the virialisation redshift. As a result, the density contrast at the time of virialisation $\Delta_{c}=\rho_{\text {cdm,inside }}\left(z_{v}\right) / \rho_{\text {cdm,outside }}\left(z_{v}\right)$ becomes strongly dependent on $z_{v}$ as well.

On the other hand, the ratio of the energy densities of dark matter and dark energy depends on the clustering properties of dark energy, but is small at the typical redshift of cluster formation, even if dark energy clusters.

We have also checked the dependence on our assumptions. For example, a more conservative assumption would be that dark energy only clusters after turnaround, i.e. once the overdensity is decoupled from the rest of the universe. Before that, the field is homogeneous. In this case, there is still a 

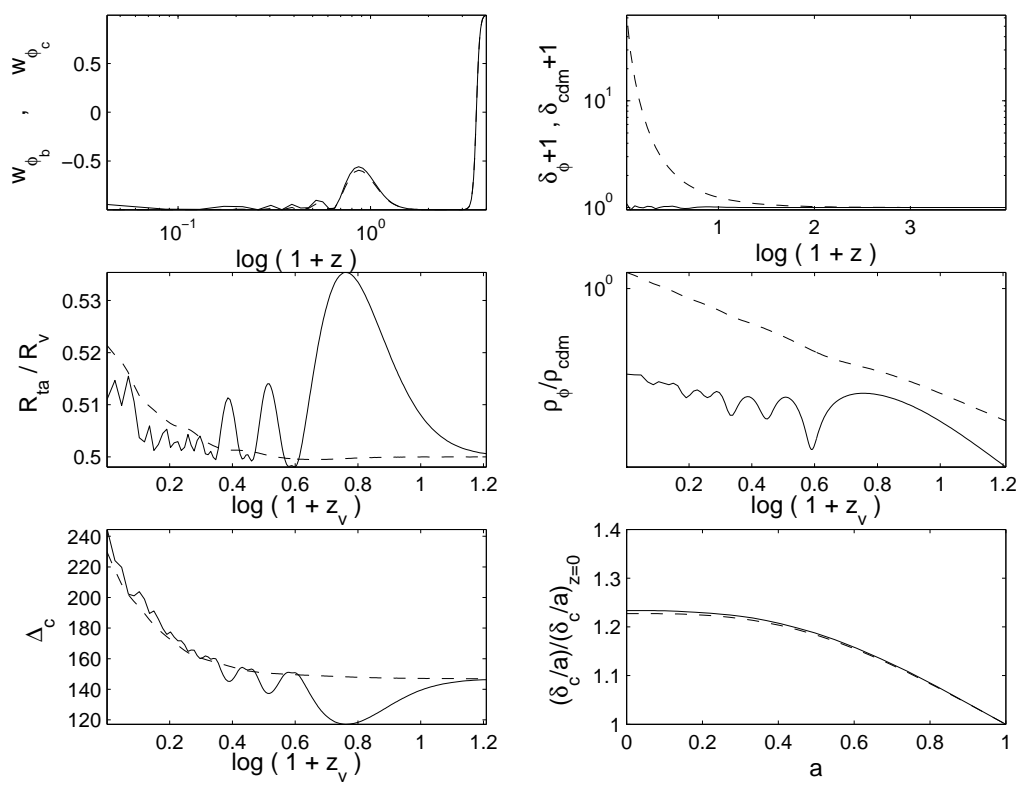

Figure 3. The same as Figure 1 but for the modified exponential potential (see text).

big difference from the homogeneous case (see our work ${ }^{4}$ for more details).

\section{Outlook}

The spherical collapse model susggests, that dark energy can have an important impact on non-linear structure formation, even if it is dynamically unimportant for most of the time during the cosmic history. We have considered two extreme cases, namely that dark energy either fully collapses together with dark matter or that dark energy is homogeneous throughout space. Its interesting to note that even if dark energy collapses with dark matter, it will stay in the linear regime (or sometimes in the quasi nonlinear regime, in which the density contrast is of order 1), whereas dark matter is in the highly non-linear regime. In fact, although dark matter enters a highly non-linear regime, the dark energy density contrast deviates from unity only slightly.

The aim of our approach was not to make predictions for structure formation, but rather to investigate if dark energy can at all have a significant impact on the details of structure formation. Clearly, our work shows that it can have, but the answer depends strongly on the details of the theory. 
Our work is rather limited, since the spherical collapse model can not predict how much dark energy will flow out of the overdensity. For this, a fully relativistic approach has to be taken, in order to calculate the amount exactly. However, our work clearly indicates that the spherical collapse model has to be used with care, when comparing models of dark energy with data.

On the other hand, our work raises some questions for future work: even if dark energy clusters strongly, the differences to a theory where it does not cluster should not be too large, since at the redshift of structure formation (i.e. $z \geq 3$ ) the differences between the theories are small. How will we be able to differentiate between the theories using only structure formation data? Note that the case of a dark energy which collapses with dark matter is rather extreme, so its likely that any signal of dark energy clustering will be even smaller, once the exact value of $\Gamma$ is known.

\section{Acknowledgements}

C.v.d.B. was supported by PPARC. D.F.M. is supported by Funda cao Ciencia e a Tecnologia.

\section{References}

1. P. J. E. Peebles and B. Rathra, Rev. Mod. Phys. 75, 559 (2003)

2. C. Wetterich, Astronomy and Astrophysics 301, 321(1995)

3. L. Amendola, Phys.Rev. D 62, 043511, (2000)

4. D. F. Mota and C. van de Bruck, Astronomy and Astrophysics 421, 71 (2004)

5. T. Barreiro, E. Copeland and N. J. Nunes, Phys. Rev. D 61, 127301 (2000)

6. P. Brax and J. Martin, Phys. Lett. B 46840 (1999)

7. A. Albrecht and C. Skordis, Phys. Rev. Lett. 84, 2076 (2000)

8. O. Lahav, P. B. Lilje, J. R. Primach and M. J. Rees, Month. Not. Roy. Astron. Soc. 251, 128 (1991) 